\title{
Da identificação à intervenção: abordagem do médico da família e comunidade na violência contra a mulher
}

\author{
From identification to intervention: a family and community doctor's approach to violence \\ agains women
}

De la identificación a la intervención: acercamiento a los médicos de família y comunitários em violência contra la mujer

Caroline Kugeratski Carneiro ${ }^{1 *}$, Juliana Gomes Margraf ${ }^{1}$, Simone Kempf Stachechem ${ }^{1}$, Ingrid Kurzawa Zwiener Ostermayer².

\section{RESUMO}

Objetivo: Propor uma reflexão acerca da temática que envolve a importância da atuação do médico da família e comunidade (MFC) em casos de violência contra a mulher. Revisão bibliográfica: Para abordagem do tema são trazidos para a discussão os fatores históricos que legitimaram a diminuição social da mulher, independente de classe social, raça ou etnia; a criação da Lei Maria da Penha, que mostra as diversas formas de agressão contra a mulher e as penalidade aplicáveis, bem como os meios jurídicos por meio dos quais a mulher pode defender-se contra atos de violência e; ainda o tipo penal chamado feminicídio, considerado como o estágio máximo de violência contra a mulher. Segue o estudo, abordando o primeiro nível de atendimento do paciente no SUS, e a importância do MFC na abordagem desses casos de violência, em especial, no que se refere a identificação e intervenção, meios através dos quais será possível dar a mulher atendimento integral, contemplando os aspectos biopsicossociais dos processos de saúde e doença. Considerações finais: A violência contra a mulher persiste, assim, mostra-se importante uma atuação multiprofissional e intersetorial, com intuito de identificar, intervir, implementar e fortalecer as ações integradas voltadas ao cuidado efetivo das vítimas.

Palavras-chave: Violência contra a mulher, Feminicídio, Medicina de família e comunidade.

\begin{abstract}
Objective: To propose a reflection about the theme that involves the importance of the family and community physician's (FCM) role in cases of violence against women. Bibliographic review: To approach the theme, the historical factors that legitimated the social diminution of women, regardless of social class, race or ethnicity are brought to the discussion; the creation of the Maria da Penha Law, which shows the several forms of aggression against women and the applicable penalties, as well as the legal means by which women can defend themselves against acts of violence, and also the criminal type called feminicide, considered the maximum stage of violence against women. The study continues, addressing the first level of patient care in the SUS, and the importance of FCM in approaching these cases of violence, especially in what refers to identification and intervention, means through which it will be possible to give the woman an integral care, contemplating the biopsychosocial aspects of the health and disease processes. Final considerations: The violence against women persists, thus, it is important a multidisciplinary and intersectoral action, in order to identify, intervene, implement and strengthen integrated actions aimed the effective care of victims.
\end{abstract}

Keywords: Violence against women, Feminicide, Family and community medicine.

1 Universidade do Contestado, Mafra - SC. *E-mail: carolinecarneiro01@gmail.com

2 Universidade Federal do Paraná, Curitiba - PR. 


\section{RESUMEN}

Objetivo: Proponer una reflexión sobre el tema que involucra la importancia del papel del médico de familia y de la comunidad (MFC) en los casos de violencia contra la mujer. Reseña bibliográfica: Para abordar el tema, se traen a la discusión los factores históricos que legitimaron la disminución social de las mujeres, independientemente de su clase social, raza o etnia; la creación de la Ley Maria da Penha, que muestra las diversas formas de agresión contra las mujeres y las penas aplicables, así como los medios legales por los que las mujeres pueden defenderse de los actos de violencia, y también el tipo penal denominado feminicidio, considerado la etapa máxima de la violencia contra las mujeres. El estudio continúa abordando el primer nivel de atención al paciente en el SUS, y la importancia de la FCM en el abordaje de estos casos de violencia, especialmente en lo que respecta a la identificación e intervención, medios a través de los cuales será posible dar a las mujeres una atención integral, contemplando los aspectos biopsicosociales de los procesos de salud y enfermedad. Consideraciones finales: La violencia contra las mujeres persiste, por lo que es importante una acción multiprofesional e intersectorial, para identificar, intervenir, implementar y fortalecer las acciones integradas dirigidas a la atención efectiva de las víctimas.

Palabras clave: Violencia contra la mujer, Feminicidio, Medicina familiar y comunitaria.

\section{INTRODUÇÃO}

A violência contra o gênero feminino possui relação com questões históricas que corrobora com a disparidade social das mulheres, ao mesmo tempo com a não capacidade do gênero masculino em adaptarse à nova realidade na qual a mulher possui independência e poder sobre si mesma. Avançar na equidade de gênero é de extrema importância para o enfrentamento do feminicídio, requer a compreensão do seu caráter relacional e desconstrução de estereótipos que atribuem às mulheres e aos homens características derivadas da história (BRASIL, 2016).

Do ponto de vista da história brasileira, essa violência é a sucessora de uma cultura que se originou em uma sociedade escravista, que foi construída a partir do modelo colonial instalado. É definido como um comportamento dirigido à mulher, simplesmente por ser mulher, com objetivo de intimidá-la e fazer com que o agressor desempenhe o papel de dominador e disciplinador. Este é um problema de saúde pública e uma violação dos direitos humanos, o que deve ser objeto de pesquisa, pois tem impacto na vida das pessoas e nos investimentos feitos pelos governos ao redor do mundo para reduzir os índices de criminalidade (MENEGHEL S, et al., 2017).

Na saúde, dentro da atenção primária (APS), caracterizada por ser o primeiro nível de atendimento ao paciente no SUS, os médicos de família e comunidade (MFC) são responsáveis pelo método clínico centrado na pessoa investigando suas queixas de maneira integrada. Além disso eles precisam conhecer profundamente os problemas de saúde que mais frequentemente ocorrem no cenário da APS para identificarem e intervirem de forma correta, como é o caso da violência contra a mulher, sendo essencial uma equipe multidisciplinar para cuidar dessas mulheres como um todo, desde aspectos físicos até emocionais (DUCAN B, et al., 2013).

As vítimas podem ser violadas de forma física, sexual e psíquica, podendo resultar em ferimentos e potencializar o desenvolvimento de queixas psicológicas como a depressão, ansiedade, e outros transtornos. Além de aumentar as chances de uso de drogas ilícitas e lícitas e substâncias psicoativas que podem resultar em sensações de prazer e bem-estar ao usuário, diminuindo, então, os sintomas e as angústias dessas mulheres (ARBOIT J, et al., 2017)

Nesse contexto, o médico e sua equipe possuem papel de extrema importância na abordagem desses casos com o intuito de prevenir episódios e reabilitar as vítimas, entretanto, essa abordagem não é tão fácil assim, ainda há um tabu em torno do tema feminicídio e muitos desafios em torno dessa intervenção. Temse que, no enfrentamento das violências o sistema judicial e o policial são os mais buscados e a saúde não tem exercido seu papel como porta de entrada para a identificação e o acolhimento nessas situações; os profissionais raramente julgam a violência contra a mulher como um problema de saúde pública (MENEGHEL S, et al., 2017). 
Diante do exposto, o presente artigo trata-se de um ensaio teórico, de base descritiva, com objetivo de propor uma reflexão acerca da temática que envolve a importância da atuação do médico da família e comunidade em casos de violência contra a mulher, justificando-se pela relevância do tema aliada à preocupação dos autores em discorrer sobre o assunto.

\section{REVISÃO BIBLOGRÁFICA}

\section{Aspectos históricos da violência doméstica contra a mulher}

Segundo a Declaração Universal dos direitos humanos, entende-se por todas as normas que regem a proteção e dignidade a todos os seres humanos, aplicadas expressamente iguais entre homens e mulheres. A violência, de acordo com a Organização Mundial da Saúde (OMS), trata-se do uso proposital da força física ou poder, contra si mesmo, contra outro indivíduo ou contra uma coletividade, que possa suceder em feridas, morte, prejuízo psicológico, desenvolvimento afetado ou a privação. Termo esse que viola de forma inerente todos os direitos humanos do cidadão (OMS, 2002; SANTOS A e WITECK G, 2016).

A violência contra as mulheres caracteriza-se pela violação da integridade de tal gênero, independente de fronteiras de classe social, raça e etnia. Tais situações são características das profundas desigualdades de poder entre homens e mulheres, que permanecem sujeitadas a esquemas de dominação e exploração. No Brasil, a violência tem grande influência de uma sociedade escravocrata, em que a mulher era vista como um objeto. Atualmente, por ser cada vez mais abordada pelos profissionais, passou a ser um problema de saúde pública (ARBOIT J, et al, 2018; NOGUEIRA S, et al., 2020).

Diante do cenário alarmante, e, por ser um fenômeno constitutivo da própria historicidade brasileira, em 7 de agosto de 2006 foi sancionada a Lei Maria da Penha (Lei $n^{\circ} 11.340$ ), sendo essa a principal defensora reconhecida hoje em nosso país, que garante o direito as mulheres; tem o intuito de prevenir, punir e erradicar todas as formas de violência contra a mulher. A lei estabelece como crime todos os atentados a integralidade da mulher, e prevê apuração por meio de inquéritos policiais remetidos ao Ministério Público e ao Conselho Nacional de Justiça, o qual trabalha em prol de divulgar e difundir a legislação e facilitar o acesso da mulher a justiça (CANAL G, et al., 2019).

De acordo com o Instituto Maria da Penha, são classificados em cinco os tipos de violência contra a mulher: física, psicológica, sexual, patrimonial e moral. A primeira é todo ato como espancamentos, apertos de braços, estrangulamento, sufocamento, ferimentos, torturas que coloque em risco a integridade ou saúde corporal da mulher. Já a segunda são ameaças, manipulações, perseguições e chantagens que causam danos emocionais à vítima limitando o direito de ir e vir tirando a liberdade da crença individual e colocando em dúvida a própria sanidade (INSTITUTO MARIA DA PENHA, 2018).

A violência sexual é todo o ato não consentido mediado por meio de intimidações, ameaças, uso de força física e coações, gerando desconforto e repulsa, como impedir o uso de métodos contraceptivos, forçar o matrimonio, gravidez ou prostituição, ou limitar/anular o exercício dos direitos sexuais e reprodutivos da mulher. O quarto, patrimonial, resulta em retenção, subtração, destruição de objetos, seja de forma parcial ou total, sendo estes pessoais, instrumentos ou documentos de trabalho, privação de bens, valores ou recursos econômicos. Por último, a violência moral, consiste em atos de calúnias, difamação ou injúria, que incidam sobre a índole da mulher e desvalorização da vítima. Diante disso, é necessário mudar a mentalidade e combater os estereótipos de gênero é uma maneira de enfrentar e não tolerar mais esse tipo de agressão (INSTITUTO MARIA DA PENHA, 2018).

\section{Feminicídio}

Arendt H (1994), filósofa alemã, defende em sua obra "Sobre Violência" que poder e violência são opostos, se a violência é praticada, há ausência de poder por parte da vítima, essa relação entre o poder e a violência está diretamente ligada a questão do feminicídio. Esse é descrito como uma forma de homicídio que tem como base a discriminação do sexo feminino, menosprezando tal gênero, constituindo a extrema violência contra a mulher, que geralmente ocorre depois de outros tipos de violência cometidos. Muitas vezes, essa prática se inicia com relatos de violência doméstica, abusos e maus-tratos (CANAL G, et al., 2019; MESSIAS E, et al., 2020). 
Diante disso, essa forma de homicídio não se refere em um só episódio separado e imprevisto; e sim, faz parte de uma sucessão de violências, caracterizando o uso da violência extrema. Muitas destas mortes podem ser "anunciadas" e evitáveis, trazendo o feminicídio como um grande indicador para situações de não equidade de gênero no grupo de pessoas, e, práticas sociais que formam este tipo de violência. Considerase parte de meios socioculturais ampliados, que passam a esfera das relações entre homens e mulheres (MENEGHEL S, et al., 2017; NOGUEIRA S, et al.,2020).

Radford J e Russel D (1992) no livrro "Femicide: the politics of woman killing", caracterizam o termo "Feminicídio" por violência extrema, que passou a ser considerado por outros territórios no final do século XX, e início do século XXI, e denunciaram as violências em que as mulheres dos Estados Unidos da América vivenciavam naquela época. Nesse sentido, as questões históricas trazem menção ao patriarcado como sendo um dos principais responsáveis pela violência como aplicação da forma de exercer autoridade e poder sobre esse gênero em questão, por meio de condutas fixadas na sociedade e dos padrões já estabelecidos para homens e mulheres que transcorre entre as gerações (CANAL G, et al., 2019).

A Organização das Nações Unidas aborda em relação a perda da vida de mulheres apenas pela condição de ser mulher, como um problema de ordem global e de saúde pública, e reforça que o Brasil é o país que possui os maiores índeces de crimes desse caráter, totalizando há 4 anos atrás mais de 1.100 vítimas. No ranking, composto por mais de 80 nações, de crimes contra a mulher, preenche o quinto lugar mesmo sendo referência na segurança desse gênero e contenha no país inúmeras leis voltadas ao combate à violência contra a mulher (CANAL G, et al., 2019).

Dados do Mapa da Violência no Brasil apresentam índices crescentes de mortes de mulheres por agressão no período de $1980-2010$, representando um aumento de mais de $100 \%$. As altas taxas estão articuladas a submissão do gênero e das condições socioeconômicas em cenários de criminalidade, criando uma nova posição de vítima para as mulheres. Esses dados fortalecem a concepção feminicídio, o qual é o crime de poder e dominação, afetando os grupos mais vulneráveis, e os contextos contribuem para entender os determinantes (MENEGHEL S, et al., 2017).

\section{Abordagem do médico da família e comunidade dentro da APS}

A atenção primária a saúde é a porta de entrada do SUS, sendo caracterizada pelo primeiro contato ao paciente. Formada por medidas de saúde que envolvem promoção de saúde, prevenção de doenças, assistência, identificação, abordagem terapêutica, reabilitação, diminuição de danos, cuidados paliativos e vigilância em saúde, desenvolvida por meio de práticas integralizadas e gestão de qualidade, executada por uma equipe multiprofissional, com os médicos de família e comunidade no centro dessas equipes (GUSSO G, et al., 2012).

A medicina de família e comunidade (MFC) teve, no Brasil, a partir de 1975, os primeiros programas de residência médica, e em 1981, especialidade denominada de Medicina Geral Comunitária por meio da Resolução da Comissão Nacional de Residência Médica (CNRM). No entanto, em 2001, foi reconhecida por Medicina da Família e Comunidade pelo CNRM e pelo Conselho Federal de Medicina (CFM), sendo definida pelo amparo à saúde de modo longitudinal, integral e abrangente para os pacientes, suas famílias e toda a comunidade adscrita (COELHO G, et al., 2019).

O Programa de Residência visa completar a formação de um médico, em termos de conhecimentos, de atitudes e habilidades necessárias para que ele utilize métodos clínicos, epidemiológicos, administrativos e sociais para proporcionar cuidados personalizados, continuados e integrais a saúde física, mental e social de uma população geograficamente definida, por meio de medidas de prevenção primária, secundária e terciárias. Além de, atenderem a maior parte da demanda de serviços de saúde da população e o restante atende por meio do encaminhamento a outros níveis do Sistema de Saúde ou a outros setores (GUSSO G, et al., 2012).

Os princípios que norteiam a especialidade de Medicina da Família e Comunidade são sobre médicos qualificados com competência no método clínico centrado na pessoa investigando suas queixas de maneira integrada que precisam conhecer profundamente os problemas de saúde que mais frequentemente ocorrem no cenário da APS, e das condições de meio ambiente ou contexto do local de trabalho. Além disso, a relação 
médico-paciente é fundamental para o desempenho do profissional, identificando sentimentos e expectativas e impactos da doença na vida pessoal, familiar, profissional e social e, com isso, procurar entender os reais motivos das pessoas às consultas (DUNCAN B, et al., 2013).

O médico de família e comunidade também presta cuidados paliativos, os quais são destinados a pessoas na fase final da vida e suas famílias, considerando que, existe muito a se fazer para diminuir o sofrimento, manter a qualidade de vida, proporcionar uma morte digna e fornecer apoio aos familiares, amenizando o sofrimento da perda e do luto. Como também, pode prestar seus cuidados em ambulatório, hospital, incluindo o setor de emergências, a domicílio ou em outros cenários, como creches, escolas, presídios e empresas (GUSSO G, et al., 2012).

\section{A abordagem do médico da família e comunidade}

A violência contra a mulher é de caráter crônico e resulta em grande impacto para a saúde física e mental dessas vítimas; percebe-se muitas queixas psíquicas como a dificuldade em dormir, irritabilidade, mudanças de humor, baixa autoestima e fragilidade emocional, podendo, então, ter prejuizo na qualidade de vida e resultar em transtornos como a ansiedade caracterizada pela preocupação e medo constantes, depressão, e ainda, o transtorno de estresse pós-traumático (ARBOIT J, et al., 2017)

Além desses transtornos, há o aumento do uso de substâncias ilícitas e lícitas e de medicamentos psicotrópicos como os antidepressivos e benzodiazepínicos que são capazes de provocar alterações no sistema nervoso central, modificando temporariamente o humor e as sensações ruins pelo alivio do medo, da dor e das angústias dessas mulheres (ARBOIT J, et al., 2017)

Diante disso, por ser um evento crônico, demanda persistência e tempo por parte dos profissionais de saúde e das vítimas para compreenderem as consequências prejudiciais de tal ato, o qual possui resultados diretos na área da saúde, já que os médicos e sua equipe multidisciplinar são os responsáveis pela assistência integral e pela reabilitação dessas mulheres (ARBOIT J, et al., 2018).

O reconhecimento dos cenários mais pertinentes da violência, por parte dos profissionais, ajuda a compreender os seus determinantes, sendo os familiares e domésticos os mais apontados. Desse modo, estimam-se fatores que podem aumentar a fragilidade dessas mulheres: diferença de idade entre os cônjuges, precariedade no nível de escolaridade, a situação matrimonial informal, as experiências anteriores da mulher em firmar a separação, condição socioeconômica, exploração, tráfico sexual e de drogas (MENEGHEL S, et al., 2017).

As mulheres que sofrem violência doméstica utilizam com mais frequência dos serviços de saúde, possuem queixas confusas que não são resolvidas, gerando sentimento de impotência nos profissionais, sendo, por vezes, desqualificadas e desacreditadas na sua demanda. Por esse motivo, a identificação dos sinais é de extrema importância para o MFC, dentre eles, pode-se citar: transtornos psiquiátricos, distúrbios alimentares, problemas ginecológicos e obstétricos, doenças sexualmente transmissíveis, gestações não planejadas com início tardio do acompanhamento do pré-natal. Além disso, no exame físico é possível visualizar lesões na região central do corpo, marcas na cabeça e pescoço sugerindo estrangulamento, e lesões nos antebraços, as quais sugerem violência (DUNCAN B, et al., 2013; GUSSO G, et al., 2012; MENEGHEL S, et al., 2017).

É essencial conhecer a família da paciente e a sua dinâmica, podendo, então, em muitos casos, identificar os casos de violência contra a mulher a partir da observação ou, também, pela relação de aproximidade entre os profissionais de saúde e a paciente. Ademais, as reuniões da equipe, as visitas domiciliares para estabelecer vínculo com as famílias e, principalmente, o conhecimento sobre a dinâmica familiar pelos agentes comunitários, e do território, juntamente com o trabalho multidisciplinar são estratégias amplamente utilizadas pelos profissionais da atenção primária (ARBOIT J, et al., 2018; MOREIRA T, et al., 2014).

Os agentes comunitários de saúde (ACS) por meio das visitas domicilares possuem importante papel no combate à violência doméstica e sua identificação pela relação direta com as famílias. Nesse sentido, conseguem analisar os fatores de risco e de vulnerabilidades, os sinais e sintomas clínicos de agressão física e psicológica, podendo, assim, auxiliar na prevenção da violência, diminuir as dificuldades no diagnóstico, e contribuir para a intervenção e abordagem desses casos (SILVA ACF, et al., 2020). 
As práticas de cuidado relacionais incluem o diálogo, o qual é apontado como um elemento-chave na abordagem da vítima da violência doméstica. São fundamentais a escuta ativa e o vínculo, orientações, trabalho em equipe, qualificação, apoio multiprofissional e intersetorial para que possam responder de modo eficaz às demandas biopsicossociais dessa população específica. $O$ atendimento deve ser prioritário, de maneira a garantir a privacidade, o estabelecimento de relação de confiança e respeito atendendo as comunicações verbais e não verbais de modo compreensivo, sem julgamentos e críticas (ARBOIT J, et al., 2018).

É importante frisar que ao assistir uma vítima de violência o profissional deve ter empatia, pois lidará com uma paciente traumatizada. Ao mesmo tempo, deve-se dar a devida importância para o impacto sobre a vida da mulher, mantendo uma postura tranquila e positiva. A abordagem psicológica correta é fundamental, a mulher pode estar assustada e silenciosa, envergonhada e confusa ou desesperada e agitada, portando, deve-se perguntar diretamente como ela está se sentindo, garantindo serviços efetivos de saúde mental, tais como psicoterapia, centros de atenção psicossocial (CAPS) e centros de referência de assistência social (CRAS) (DUNCAN B, et al., 2013).

Desse modo, é primordial que os profissionais da saúde de diversas especialidades troquem informações, aprendizagens e sabedorias em relação à violência doméstica contra as mulheres, buscando o amadurecimento das ações que possibilitem o cuidado integral e longitudinal a essas vítimas (ARBOIT J, et al., 2018).

\section{Desafios na abordagem: identificação e intervenção}

A falta de conhecimento de meios de notificação nesses casos de violência causa transtornos psicológicos e questionamentos nos profissionais da atenção primária. Esses fatores, em conjunto com a sobrecarga de trabalho, possuem como consequência a não observação da complexidade da situação e a não urgência de resolução, contribuindo, assim, com a persistência da subnotificação dos casos e a não visibilidade desta problemática (LEITE A e FONTELLA B, 2019).

Em relação a abordagem da violência, existe certa fragilidade e limitado preparo dos profissionais. A atuação das equipes mostra a tendência a "medicalizar" a violência, com atitudes prescritivas e intervencionistas em relação à família, não é rara a adoção de atitudes de omissão frente aos casos, não os tomando como objeto de seu trabalho, tornando-se, apenas, de caráter pontual e emergencial. A falta de preparo da equipe potencializa os sentimentos de medo, angústia e impotência e expõe às consequências de ações inadequadas ou pouco resolutivas (MOREIRA T, et al., 2014).

Outro fator que dificulta a atuação dos profissionais da área da saúde é a insuficiência ou inexistência de serviços especializados e equipamentos sociais como casas de passagem que possam acolher e proteger essas mulheres. Para o profissional da APS, que está na ponta porta de entrada do sistema traz-se um sentimento de solidão e desamparo, sentem-se despreparados. Além disso, os médicos não se sentem capazes de abordar sozinhos a questão, pressupondo que o trabalho em equipe poderia tornar esse cuidado de fácil execução (ARBOIT J, et al., 2018; MENEGHEL S, et al., 2017).

\section{CONSIDERAÇÕES FINAIS}

$\mathrm{Na}$ historicidade brasileira, identifica-se a presença de uma sociedade patriarcal, na qual, as mulheres foram preparadas para estabelecer uma relação de submissão e obediência diante de seus parceiros. Mesmo com a criação de leis e políticas públicas para o amparo dessas mulheres, a violência doméstica ainda persiste, em índices relativamente altos. Diante do exposto, a presente reflexão aponta que os enfrentamentos das situações de violência necessitam de uma abordagem multiprofissional e intersetorial, pressupondo a implementação e fortalecimento de ações integradas voltadas ao cuidado efetivo das vítimas, com intuito a promoção de qualidade de vida e saúde, além de nortear o planejamento e criação de políticas públicas voltadas à causa. Nesse sentido, entende-se que os profissionais da atenção primária em saúde, devem atuar de modo a privilegiar o desenvolvimento de estratégias de identificação e intervenção no que se refere às situações de violência doméstica contra mulheres, visando um cuidado integral, contemplando aspectos biopsicossociais dos processos de saúde e doença. 


\section{REFERÊNCIAS}

1. ARBOIT J, et al. Atenção à saúde de mulheres em situação de violência: desarticulação dos profissionais em rede. Revista da Escola de Enfermagem da USP, 2017; 51.

2. ARBOIT J, et al. Violência doméstica contra mulheres rurais: práticas de cuidado desenvolvidas por agentes comunitários de saúde. Saude soc., São Paulo, 2018; 27(2): 506-5017.

3. ARENDT H. Sobre a Violência. Rio de Janeiro: Relume-Dumará, 1994.

4. BRASIL. Lei no 10.778 , de 24 de novembro de 2003. Estabelece a notificação compulsória, no território nacional, do caso de violência contra a mulher que for atendida em serviços de saúde públicos ou privados. Brasília, 2003. Disponível em: http://www.planalto.gov.br. Acesso em: 10 abril. 2021

5. BRASIL. Manual do Ministério da Saúde. 2016. Protocolos da Atenção Básica: saúde das mulheres. Brasília (DF): Ministério da Saúde. Disponível em: https://bvsms.saude.gov.br. Acesso em: 10 abril. 2021

6. BRASIL. Ministério da Saúde. Gabinete do Ministro. Portaria № 737, de 16 de Maio de 2001. Brasília, 2001. Disponível em: https://bvsms.saude.gov.br. Acesso em: 10 abril. 2021

7. CANAL G, et al. Feminicídio: o gênero de quem mata e de quem morre. Serviço Social em Revista. 21.333. 10.5433/1679-4842. 2019; 21(2): 333.

8. COELHO G, et al. A prática da Medicina de Família e Comunidade no Brasil: contexto e perspectivas, 2019.

9. DIAS I. Violência doméstica e justiça: respostas e desafios. Sociologia: Revista do Departamento de Sociologia da FLUP, 2010; 20: 245-262.

10. DUNCAN B, et al. Medicina ambulatorial: condutas de atenção primária baseadas em evidências. Porto Alegre: Artmed, 2013.

11. FERRAZ M, et al. O cuidado de enfermagem a vítimas de violência doméstica. Cogitare Enfermagem, [s.I.],2009; 14(4): 756-759. Universidade Federal do Paraná.

12. FONSECA D, et al. Violência doméstica contra a mulher: realidades e representações sociais. Psicol. Soc., Belo Horizonte, 2012; 24(2): 307-314.

13. GUSSO G, et al. Tratado de medicina de família e comunidade: princípios, formação e prática. Porto Alegre: Artmed, 2012.

14. VIOLÊNCIA DOMÉSTICA, 2018. In: INSTITUTO MARIA DA PENHA. Disponível em: https://www.institutomariadapenha.org.br/ Acesso em: 10 abril. 2021

15. LEITE A e FONTANELLA B. Violência doméstica contra a mulher e os profissionais da APS. Revista Brasileira de Medicina de família e comunidade. 2019; 10.5712

16. MAIA C. Sobre o (des)valor da vida: feminicídio e biopolítica. História, Assis/Franca, 2019; 38: e2019052. Epub Dec 02, 2019.

17. MENEGHEL S, et al. Feminicídios: conceitos, tipos e cenários. Ciênc. Saúde coletiva, Rio de Janeiro, 2017; 22(9): 3077-3086.

18. MESSIAS E, et al. Feminicídio: Sob a perspectiva da dignidade da pessoa humana. Rev. Estud. Fem., Florianópolis, 2020; 28(1): e60946.

19. MOREIRA T, et al. A construção do cuidado: o atendimento às situações de violência doméstica por equipes de Saúde da Família. Saude soc., São Paulo, 2014; 23(3): 814-820.

20. NOGUEIRA S, et al. Aportes conceituais sobre o fenômeno do feminicídio. Rev Outros Tempos: Feminilidades e Masculinidades em Focos, 2020.

21. PIOSIADLO L, et al. Subalternidade de gênero: refletindo sobre a vulnerabilidade para violência doméstica contra a mulher. Esc. Anna Nery, Rio de Janeiro, 2014; 18(4): 728-733.

22. RADFORD J, RUSSELL DEH. Femicide: the politics of woman killing. Great Britain, Open University Press, 1992.

23. SANTOS A, WITECK G. Violência doméstica e familiar contra a mulher. Unisc: trabalhos científicos internacionais. Rio Grande do Sul, 2016.

24. SILVA ACF, et al. Violência sexual por parceiro íntimo identificada em Unidade Básica do PSF. Revista Nursing, 2020; 23(263): 3705-3709. 